\title{
Visceral fat inflammation and fat embolism are associated with lung's lipidic hyaline membranes in subjects with COVID-19
}

\author{
Georgia Colleluori (iD ${ }^{1,7}$, Laura Graciotti ${ }^{2,3,7}$, Mauro Pesaresi ${ }^{4}$, Angelica Di Vincenzo ${ }^{1}$, Jessica Perugini ${ }^{1}$, Eleonora Di Mercurio ${ }^{1}$, \\ Sara Caucci ${ }^{3}$, Patrizia Bagnarelli ${ }^{3}$, Cristina M. Zingaretti ${ }^{1}$, Enzo Nisoli $\mathbb{D}^{5}{ }^{5}$, Stefano Menzo ${ }^{3}$, Adriano Tagliabracci ${ }^{4}$, Annie Ladoux ${ }^{6}$, \\ Christian Dani ${ }^{6}$, Antonio Giordano ${ }^{1}$ and Saverio Cinti $\mathbb{D}^{1 凶}$
}

(c) The Author(s), under exclusive licence to Springer Nature Limited 2022

BACKGROUND: Preliminary data suggested that fat embolism could explain the importance of visceral obesity as a critical determinant of coronavirus disease-2019 (COVID-19).

METHODS: We performed a comprehensive histomorphologic analysis of autoptic visceral adipose tissue (VAT), lungs and livers of 19 subjects with COVID-19 (COVID-19+), and 23 people without COVID-19 (controls). Human adipocytes (hMADS) infected with SARS-CoV-2 were also studied.

RESULTS: Although there were no between-group differences in body-mass-index and adipocytes size, a higher prevalence of CD68+ macrophages among COVID-19+ VAT was detected $(p=0.005)$ and accompanied by crown-like structures presence, signs of adipocytes stress and death. Consistently, human adipocytes were successfully infected by SARS-CoV-2 in vitro and displayed lower cell viability. Being VAT inflammation associated with lipids spill-over from dead adipocytes, we studied lipids distribution by ORO. Lipids were observed within lungs and livers interstitial spaces, macrophages, endothelial cells, and vessels lumen, features suggestive of fat embolism syndrome, more prevalent among COVID-19+ $(p<0.001)$. Notably, signs of fat embolism were more prevalent among people with obesity $(p=0.03)$ independently of COVID-19 diagnosis, suggesting that such condition may be an obesity complication exacerbated by SARS-CoV-2 infection. Importantly, all infected subjects' lungs presented lipids-rich (ORO+) hyaline membranes, formations associated with COVID-19-related pneumonia, present only in one control patient with non-COVID19-related pneumonia. Importantly, transition aspects between embolic fat and hyaline membranes were also observed.

CONCLUSIONS: This study confirms the lung fat embolism in COVID-19+ patients and describes for the first time novel COVID-19related features possibly underlying the unfavorable prognosis in people with COVID-19 and obesity.

International Journal of Obesity (2022) 46:1009-1017; https://doi.org/10.1038/s41366-022-01071-w

\section{INTRODUCTION}

Since December 2019, the severe acute respiratory syndrome coronavirus 2 (SARS-CoV-2), responsible for the development of coronavirus disease 2019 (COVID-19), has spread globally, resulting in a worldwide health crisis that caused over five million deaths [1]. The lung is a crucial target organ not only due to the severe bilateral pneumonia observed in $15-30 \%$ of hospitalized patients $[2,3]$ but also because it is the site from which the infection spreads to blood vessels, heart, gut, brain, and kidneys [4]. Published data support interstitial fibrosis with alveolar hyaline membrane (HM) formation as the main underlying histopathologic event responsible for pneumonia and acute respiratory syndrome distress $[5,6]$. The reasons for HM bilateral expression, histogenesis, and sudden clinical appearance during COVID-19 early stages are not completely understood [7].
The severity of COVID-19 is strictly associated with the presence of comorbidities [8]; while obesity alone is responsible for $20 \%$ of COVID-19 hospitalizations, obesity in combination with type 2 diabetes and hypertension accounts for 58\% [9]. Obesity and impaired metabolic health are in fact strongly associated with COVID-19 unfavorable prognosis and pose also young patients at higher risks $[10,11]$. Significantly, visceral obesity increases the risk of COVID-19-related complications, independently of age, gender, body mass index [12], total and subcutaneous adipose tissue areas [13-16]. Visceral obesity is, in fact, strongly associated with chronic low-grade inflammation, blood hypercoagulability, impaired metabolic health, and higher risk of cardiovascular events, all risk factors for COVID-19 severity [8, 11, 16-18]. Visceral adipose tissue (VAT) excessive expansion is paralleled by adipocytes hypertrophy, death, and lipids spill-over, phenomena resulting in macrophages

\footnotetext{
${ }^{1}$ Center for the Study of Obesity, Department of Experimental and Clinical Medicine, Marche Polytechnic University, Via Tronto $10 \mathrm{~A}$, Ancona, Italy. ${ }^{2}$ Section of Experimental Pathology, Department of Clinical and Molecular Sciences, Marche Polytechnic University, Via Tronto 10A, Ancona, Italy. ${ }^{3}$ Section of Microbiology, Department of Bioscience and Public Health, Marche Polytechnic University, Via Tronto 10A, Ancona, Italy. ${ }^{4}$ Section of Legal Medicine, Department of Bioscience and Public Health, Marche Polytechnic University, Via Tronto 10A, Ancona, Italy. ${ }^{5}$ Center for Study and Research on Obesity, Department of Medical Biotechnology and Translational Medicine, University of Milan, Via Vanvitelli 32, Milan, Italy. ${ }^{6}$ Université Côte d'Azur, CNRS, Inserm, iBV, Faculté de Médecine, 06107 Cedex 2 Nice, France. ${ }^{7}$ These authors contributed equally: Georgia Colleluori, Laura Graciotti. ${ }^{凶}$ email: cinti@univpm.it
}

Received: 26 October 2021 Revised: 5 January 2022 Accepted: 12 January 2022 Published online: 26 January 2022 
infiltration, crown-like structures (CLS) development and inflammation, in turn contributing to the obesity-related complications [19-21]. The elevated adipocytes ACE2 expression in obesity [22], receptor exploited by SARS-CoV-2 for cell entry, has been often speculated as a possible pathophysiological mechanism responsible for obesity-related COVID-19 severity [8, 23, 24]. However, although obesity has been strongly associated with COVID-19 severity, original articles comprehensively analyzing adipose tissue samples belonging to subjects with COVID-19 and providing direct evidence of SARS-CoV-2 infection are lacking [16]. In our preliminary study, we described the presence of embolic fat in the lung of patients with obesity died from COVID-19 and we hypothesized that such histopathologic hallmark could be due to adipocytes stress induced by SARS-CoV-2 infection [23]. In the present study, we performed for the first time a comprehensive histomorphological assessment of VAT, lung, and liver autoptic samples belonging to subjects with (COVID-19+) and without (controls) COVID-19, and specifically, we focused on tissue lipid distribution. We observed novel SARS-CoV-2-related histopathological features, i.e., VAT inflammation, signs of fat embolism in different organs, and HM of lipidic nature in the lung, possibly contributing to the severity of COVID-19 among subjects with visceral obesity.

\section{RESULTS}

Autoptic VAT, lung and liver samples belonging to 49 subjects were collected and screened to be included in the study. Forty-two subjects were considered suitable for the study (i.e., goodpreservation for the histomorphological analyses), 19 of which died due to COVID-19-related bilateral pneumonia (COVID-19+ group), while the remaining 23 died for different reasons (control group). Subject characteristics, including gender, age, BMl, comorbidities, and cause of death, are reported in Supplementary Tables 1 and 2. SARS-CoV-2 infection was assessed by RT-qPCR performed on nasal pharyngeal or pharyngeal swab samples. Study population mean age was $65.0 \pm 14.3$ years old, BMI was $29.0 \pm 5.4 \mathrm{~kg} / \mathrm{m}^{2}$ with $35.7 \%$ of patients suffering from obesity $\left(\mathrm{BMI} \geq 30.0 \mathrm{~kg} / \mathrm{m}^{2}\right)$, and $45.2 \%$ being overweight $\left(\mathrm{BMI} \geq 25.0 \mathrm{~kg} / \mathrm{m}^{2}\right)$. In total, $35 \%$ of the population was composed of women $(n=15)$. There were no significant differences in mean age (COVID-19+: $69.5 \pm 11.0$ vs controls: $61.0 \pm$ 16.0 years old; $p=0.09$ ) and BMI (COVID-19+: $30.0 \pm 5.0$ vs controls: $28.1 \pm 5.6 \mathrm{~kg} / \mathrm{m}^{2} ; p=0.62$ ) between our study groups.

Unequivocal signs of chronic, low-grade inflammation in both COVID-19+ and control subjects with a BMI $\geq 25.0 \mathrm{~kg} / \mathrm{m}^{2}$ were observed in VAT samples (Fig. 1A). However, although there were no between-group differences in BMI and VAT adipocytes size (Fig. 1B), higher prevalence of $\mathrm{CD}^{+} 8^{+}$macrophages (Fig. 1C) and a trend for higher presence of CLS (Fig. 1D) were evidenced in COVID-19+ compared to controls, suggesting higher SARS-CoV-2-induced VAT inflammation. Other inflammatory cells were represented mainly by lymphocytes, but their number was negligible in all investigated cases.

We then assessed whether the higher VAT inflammation in COVID-19+ was associated with adipocyte death. Perilipin 1 (PLIN1) immunohistochemistry is a reliable method for identifying and quantifying dead adipocytes [19, 25]. However, in the present study, all samples display PLIN1 negative adipocytes, probably due to the autoptic nature of the specimens. We thus performed a morphologic and ultrastructural study to assess VAT adipocyte

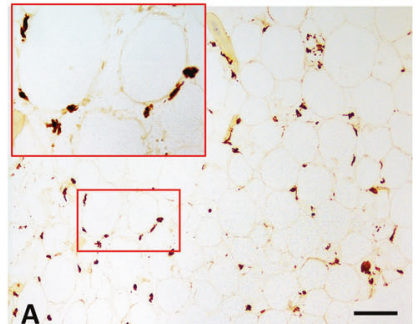

iA.

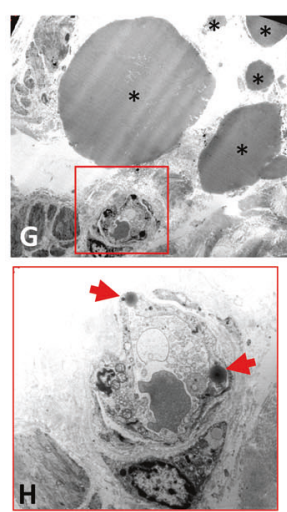

*

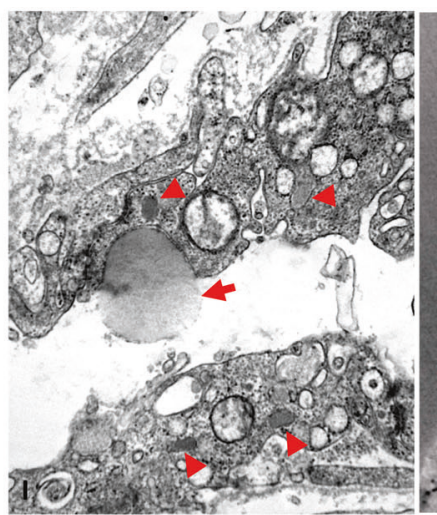

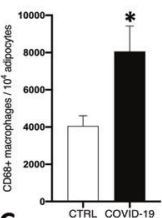

C

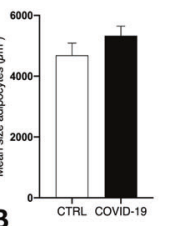

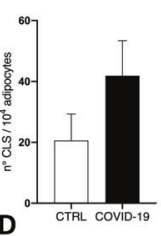
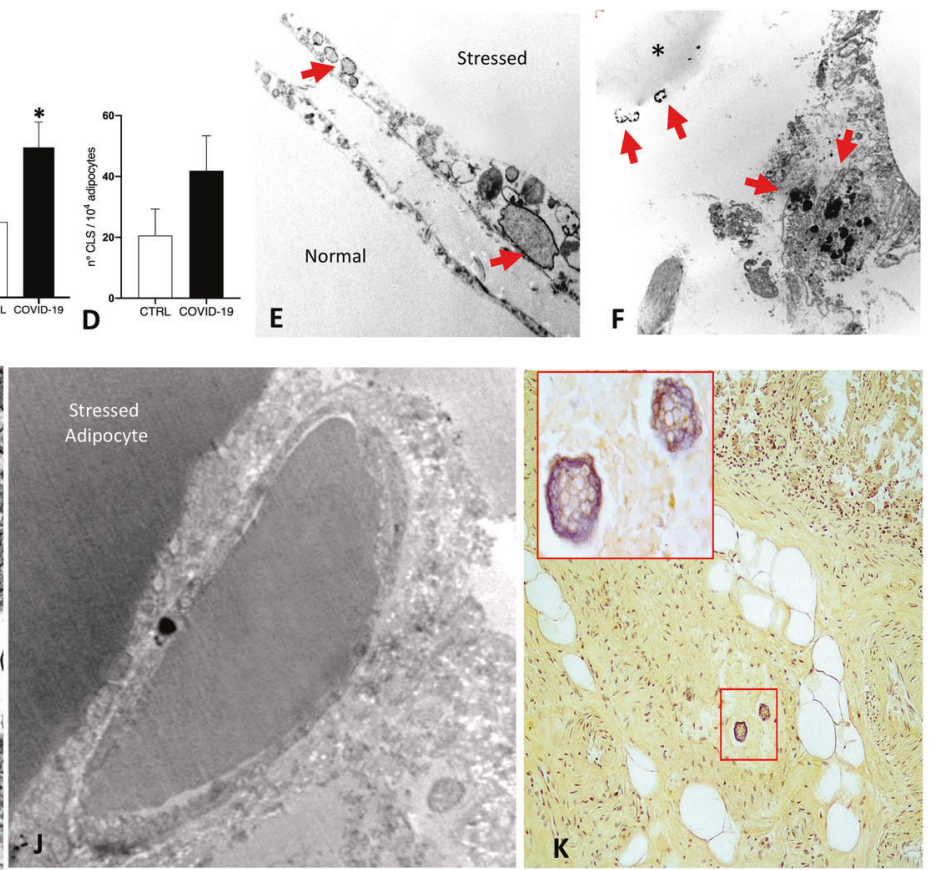

Fig. 1 Visceral adipose tissue inflammation and fat embolism in subjects with COVID-19. A Light microscopy (LM): representative immunohistochemistry of visceral adipose tissue infiltrated by CD68+ macrophages (in brown); inset shows an enlargement of the squared area. B Visceral adipose tissue adipocytes area, C number of CD68+ macrophages per $10^{4}$ adipocytes, and $\mathbf{D}$ number of crown-like structures (CLS) per $10^{4}$ adipocytes in COVID-19+ vs control subjects. Asterisk $\left(^{*}\right)$ indicates $p<0.05$. E Transmission electron microscopy (TEM): normal adipocyte adjacent to a stressed adipocyte showing dilated endoplasmic reticulum (arrows). F TEM: dead adipocytes and interstitial free lipid droplets $(*)$; arrows indicate adipocytes remnants. G TEM: free lipid droplets of variable size were frequently found in COVID-19 subjects (asterisks). $\mathbf{H}$ Enlargement of the squared area in G showing lipid droplets inside endothelial cells (arrows). I TEM: enlargement of a capillary from a COVID-19+ subject showing a lipid droplet extruding into the capillary lumen (arrow), note the abundant Weibel-Palade bodies denoting increased blood hypercoagulability (arrowheads). J TEM: a capillary filled with embolic fat near a stressed adipocyte. K LM: mesenteric fat sample showing lipid-rich embolic material in a vein (squared area, enlarged in inset). Morphometric data are expressed as means \pm SE. Scale bar: $\mathbf{A}=100 \mu \mathrm{m}, \mathbf{E}=0.8 \mu \mathrm{m}, \mathbf{F}=2.5 \mu \mathrm{m}, \mathbf{G}=10 \mu \mathrm{m}, \mathbf{H}=3 \mu \mathrm{m}, \mathbf{I}=1.5 \mu \mathrm{m}, \mathbf{J}=0.8 \mu \mathrm{m}, \mathbf{K}=35 \mu \mathrm{m}$. 

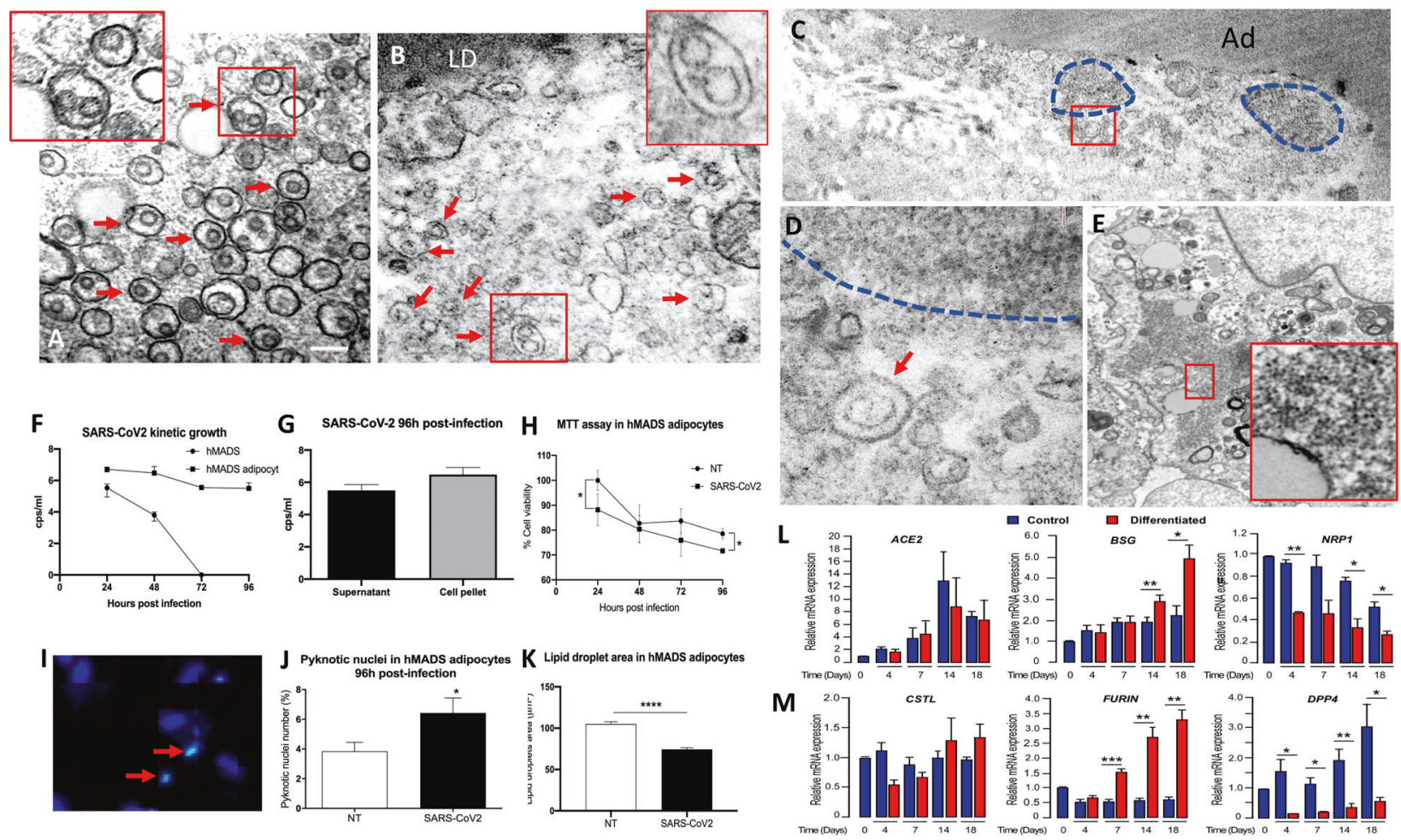

Fig. 2 SARS-CoV-2 in visceral adipose tissue and hMADS. A Transmission electron microscopy (TEM): VeroE6 infected cell showing several virions into the rough endoplasmic reticulum (RER), some indicated by arrows. Inset: enlargement of squared area. B TEM: adipocyte from the visceral adipose tissue (VAT) depot of a COVID-19+ subject showing several virions into RER (arrows). Inset: enlargement of squared area. C TEM: VAT of a COVID-19+ subject showing an adipocyte (Ad) with two large ribosome-like clusters (dotted lines) in the cytoplasm. D Enlargement of squared area in C shows a ribosome-like cluster and a virion-like structure into the dilated RER (arrow). E TEM: SARS-CoV-2infected VeroE6 cells showing a ribosome-like cluster (squared area), enlarged in the inset. F Kinetic of SARS-CoV-2 infection in undifferentiated and differentiated hMADS. SARS-CoV-2 genomic RNA detected in the supernatant at different time points expressed as copies $(\mathrm{cps}) / \mathrm{ml}$. G SARS-CoV-2 quantification in supernatant and cell pellets of hMADS infected cells. $\mathbf{H}$ The MTT viability assay in hMADS adipocytes shows lower cell viability in the infected compared to the uninfected cells at 24- and 96-h post-infection $(p<0.05)$. I Hoechst nuclear staining showing pyknotic nuclei (arrows). J Percentage of pyknotic nuclei in hMADS at $96 \mathrm{~h}$ post-infection compared to uninfected controls $(p<0.05)$. K Lipid droplets average area $\left(\mu \mathrm{m}^{2}\right)$ in differentiated hMADS $96 \mathrm{~h}$ post-infection compared to uninfected controls $(p<$ $0.0001)$. Expression of putative SARS-CoV-2 receptors $\mathbf{L}$ or proteases $\mathbf{M}$ assessed by RT-qPCR and normalized for the expression of $36 B 4$ mRNA. Expressions were measured in cells that received (red bars) or did not receive (blue bars) the differentiation cocktail for the indicated number of days. The means \pm SEM were calculated from three independent experiments (ACE2, BSG, NRP1, CSTL) or four independent experiments (FURIN, DPP4), with determinations performed in duplicate $\left({ }^{*} p<0.05,{ }^{* *} p<0.01\right)$. Scale bar: A, B $=200 \mathrm{~nm}, \mathbf{C}=500 \mathrm{~nm}, \mathbf{D}=100 \mathrm{~nm}$, $\mathbf{E}=180 \mathrm{~nm}, \mathbf{F}=120 \mu \mathrm{m}, \mathbf{G}=70 \mu \mathrm{m}, \mathbf{H}=5 \mu \mathrm{m}$.

stress and death. Electron microscopy showed frequent signs of adipocytes death in the proximity of CLS in both COVID-19+ and control individuals with a $\mathrm{BMI} \geq 25 \mathrm{~kg} / \mathrm{m}^{2}$, a finding consistent with previous studies documenting obesity-related adipocyte death [26]. Furthermore, the VAT of COVID-19+ was rich in stressed and dead adipocytes (75\% in $~ 120$ mature adipocytes; Fig. 1E, F) also in areas lacking CLS and seemingly normal at light microscopy (LM). In line with the observed widespread death, cell remnants were evident in closed proximity of dying adipocytes, while free lipid droplets were often found in fat interstitial spaces (Fig. 1F, G). Notably, large lipid vacuoles were frequently observed: (1) inside endothelial cells belonging to capillaries adjacent to free lipid droplets (Fig. 1H, I); (2) extruding from endothelial cells into the capillary lumen (Fig. 11); (3) in the lumen of VAT capillaries (Fig. 1J); (4) in macrophages near interstitial free lipid droplets (data not shown). In addition, several clusters of lipid-rich structures were found in the lumen of $\sim 11 \%$ of the venules observed in the mesenteric fat samples (total venules examined 175; Fig. 1K). In summary, the in-depth ultrastructural analyses of COVID-19+ VAT autoptic samples revealed the widespread presence of free lipid droplets inside the capillary lumen, likely derived from the dead adipocytes; all these features underline a condition able to generate fat embolism syndrome [27].
We then aimed at assessing whether the observed VAT alterations were associated with SARS-CoV-2 presence in the tissue or if they were a consequence of the systemic inflammation. Although SARS-CoV-2 ability to infect human adipose tissue has been frequently speculated $[8,13,18,23]$, direct evidence of such phenomenon are scarce in the literature $[16,28,29]$. While SARSCoV-2 genomic RNA, nucleocapsid and spike proteins were not detectable in VAT samples of patients affected by COVID-19, viruslike structures with morphology and size resembling those present in SARS-CoV-2-infected VeroE6 cells (Fig. 2A) were quite often found in the cytoplasm of stressed adipocytes (Fig. 2B). Furthermore, the presence of ribosome-like clusters described in virus-infected cells [30] was evident in visceral adipocytes of individuals with COVID-19, although rarely, (Fig. 2C, D) and frequently in SARS-CoV-2-infected VeroE6 cells (24-48 h postinfection) (Fig. 2E). In addition typical sign of several pathological conditions of viral infection, such as confronting cisternae, ribosome lamella complex and annulate lamellae [31], were observed in $\sim 25 \%$ of VAT cells belonging to subjects with COVID19 (Supplementary Fig. 1A-D) and in $45 \%$ of SARS-CoV-2infected VeroE6 cells (Supplementary Fig. 1E), but not in their uninfected respective controls. Next, to provide direct evidence of SARS-CoV-2 ability to infect human adipocytes, leading to cell 

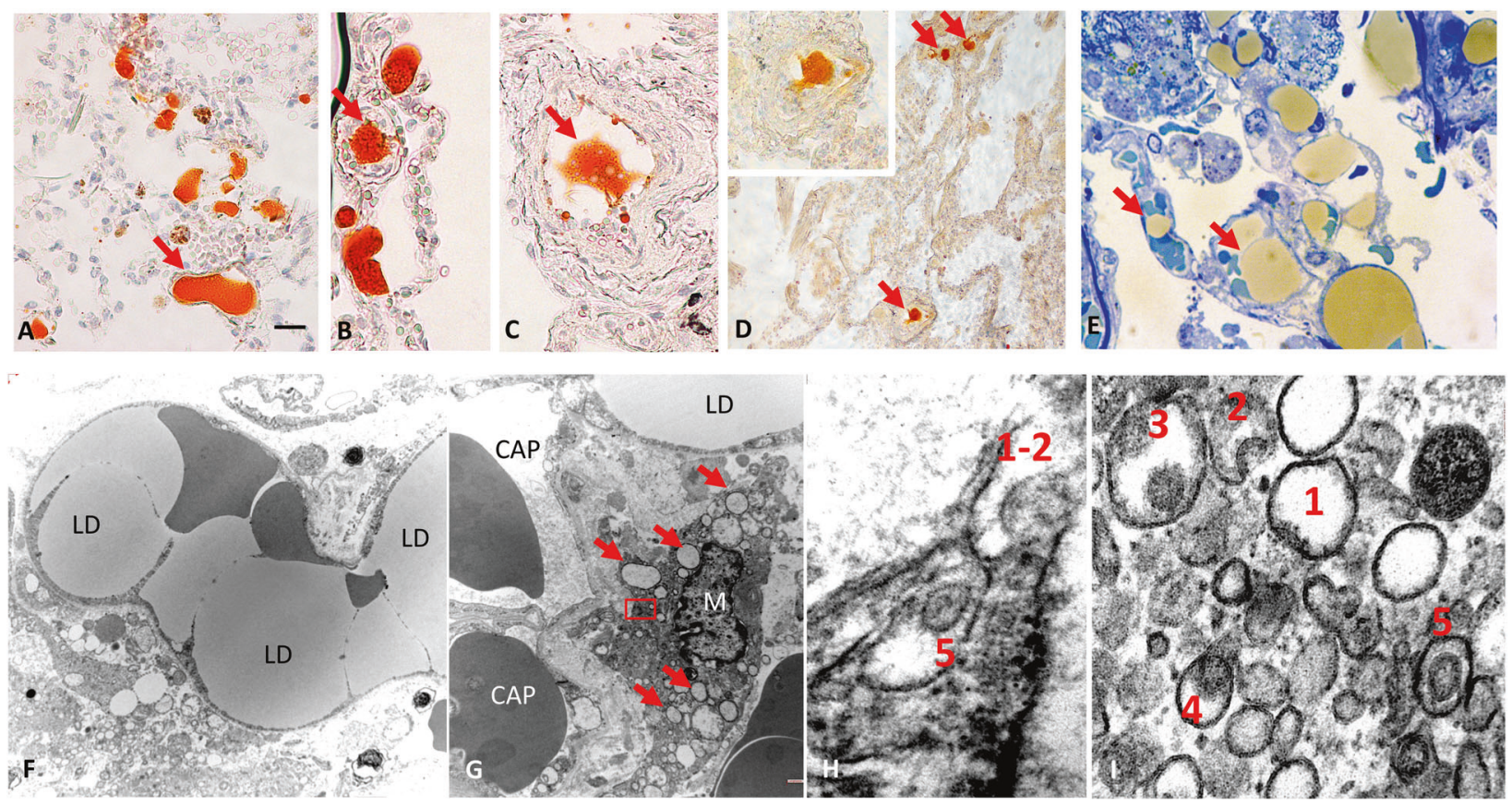

Fig. 3 Embolic lipid droplets and SARS-CoV-2 virions in the lung of subjects with COVID-19. A-D Light microscopy (LM): representative histochemistry for fat (Oil Red O) showing the lipid nature of vacuoles (orange-red) in the vascular lumen (arrows) and lung septa of different COVID-19+ subjects. E LM: resin embedded, toluidine-blue stained tissue. Large free lipid droplets (yellow) are evident in the capillaries lumen in alveolar septa (arrows). F Transmission electron microscopy (TEM): showing lipid droplet (LD) into an alveolar septum mixed with erythrocytes. G TEM: alveolar macrophage (M) in a COVID-19 subject. Note: diffuse dilated rough endoplasmic reticulum (RER) denoting cellular stress (arrows) H TEM: enlargement of the squared area in $\mathbf{G}$ showing two virions at stages 1-2 and 5 of the reproductive cycle into the dilated RER similar to what observed in I TEM: (1 to 5) stages of the reproductive cycle of SARS-CoV-2 virions in VeroE6 infected cells. Reference in the main text. Scale bar: $\mathbf{A}-\mathbf{C}=20 \mu \mathrm{m}, \mathbf{D}=140 \mu \mathrm{m}, \mathbf{E}=8 \mu \mathrm{m}, \mathbf{F}=1.5 \mu \mathrm{m}, \mathbf{G}=1 \mu \mathrm{m}, \mathbf{H}=70 \mathrm{~nm}, \mathbf{I}=65 \mathrm{~nm}$.

stress and death, we infected differentiated human multipotent adipocytes (hMADS) (Fig. 2F-H), and evaluated SARS-CoV-2 kinetics in vitro. The growth kinetics of SARS-CoV-2 was determined as viral load (copies $/ \mathrm{ml}$ ) in the supernatants collected after 24-, 48-, 72- and 96-h post-infection (Fig. 2F). Though SARSCoV-2 genomic RNA was detectable in both differentiated and undifferentiated hMADS at the first time points post-infection (24 and $48 \mathrm{~h}$ ), it could be detected only in mature adipocytes at later time points (72 and $96 \mathrm{~h}$ ) (Fig. 2F). Consistently, SARS-CoV-2 genomic RNA was also detected in the hMADS adipocytes pellet after 96-h of infection (Fig. 2G). Importantly, the infected hMADS adipocytes displayed lower cell viability (Fig. 2H), higher prevalence of pyknotic nuclei (Fig. 2I-L), and smaller lipid droplet size-suggestive of cell delipidation and stress-compared to the uninfected controls (Fig. 2K). Furthermore, in line with these data, evidence of increased material extrusion from infected cells compared to uninfected controls was observed by LM $(p<0.05)$, strongly suggesting massive cell delipidation induced by SARSCoV-2 (Supplementary Fig. 1F-H). We hence performed a timecourse analysis of hMADS expression of putative SARS-CoV-2 receptors (Fig. 2L) and proteases (Fig. 2M) in the presence or absence of the adipogenic differentiation cocktail (at 4, 7, 14, and 18 days). ACE2 receptor was expressed at very low levels in both differentiated and undifferentiated hMADS, even though we used specifically designed primers holding a $100.92 \%$ efficiency. On the other side, the BASIGIN receptor was preferentially detected in differentiated hMADS which displayed an increased expression after 14 days. The receptor NEUROPILIN 1 was expressed by undifferentiated cells. Concerning proteases expression, while differentiated hMADS expressed the protease FURIN, the undifferentiated ones preferentially expressed DPPIV. The expression of CATHEPSIN $L$ did not differs between the two conditions, while we did not detect TMPRSS2 in both differentiated and undifferentiated hMADS (data not shown).
Given our previous observation [23] and the widespread lipid droplets presence in the capillary lumen of VAT and in some mesenteric adipose depots, we studied lipid distribution in lung samples employing Oil Red O staining (ORO, i.e., the lipid-specific histochemistry). Lipids were evidenced within lungs alveolar septa, interstitial spaces, endothelial cells, vessel lumen, and in alveolar and interstitial macrophages (Fig. 3A-D), all features confirmed by light and electron microscopy (Fig. 3E, F) and suggestive of fat embolism.

Fat embolism in the lungs was not exclusive to COVID-19+ but was more prevalent among them than in controls (100\% vs $53 \%$; $p<0.001)$. Signs of fat embolism were in fact more prevalent among individuals with obesity than in those with a $\mathrm{BMI} \leq 30 \mathrm{~kg} /$ $\mathrm{m}^{2}(93 \%$ vs $63 \%, p=0.03)$, independently of COVID-19 diagnosis. Consistently, all subjects with type 2 diabetes had fat embolism. Of note, electron microscopy observations revealed several structures with size and morphology compatible with those of SARS-CoV-2 viruses [6] in pneumocytes, endothelial cells and macrophages, the last of which displayed disseminated, dilated endoplasmic reticulum denoting cellular stress $[26,32]$ and signs of virus presence only in subjects with COVID-19 (Fig. 3G, H). Furthermore, we rarely evidenced (in 2 out of 20 lung sections obtained from 8 COVID-19+) virions at early and late stages of their reproductive cycle [33] into the dilated endoplasmic reticulum (Fig. $3 \mathrm{H}$ ) comparable with those rarely revealed in infected VeroE6 (Fig. 3I) (5 out of $\sim 250$ cells). Importantly, septal capillaries very often contained large amounts of fibrin, with some of them lined by thrombotic material only in the lungs of COVID$19+$ individuals (data not shown). Several Weibel-Palade bodies, signs of activated coagulative phenomena [31], were also observed in most of the capillary endothelial cells of subjects with COVID-19 (data not shown).

Unexpectedly, the used lipid-specific histochemistry technique evidenced that all alveolar structures reminiscent of HM were 

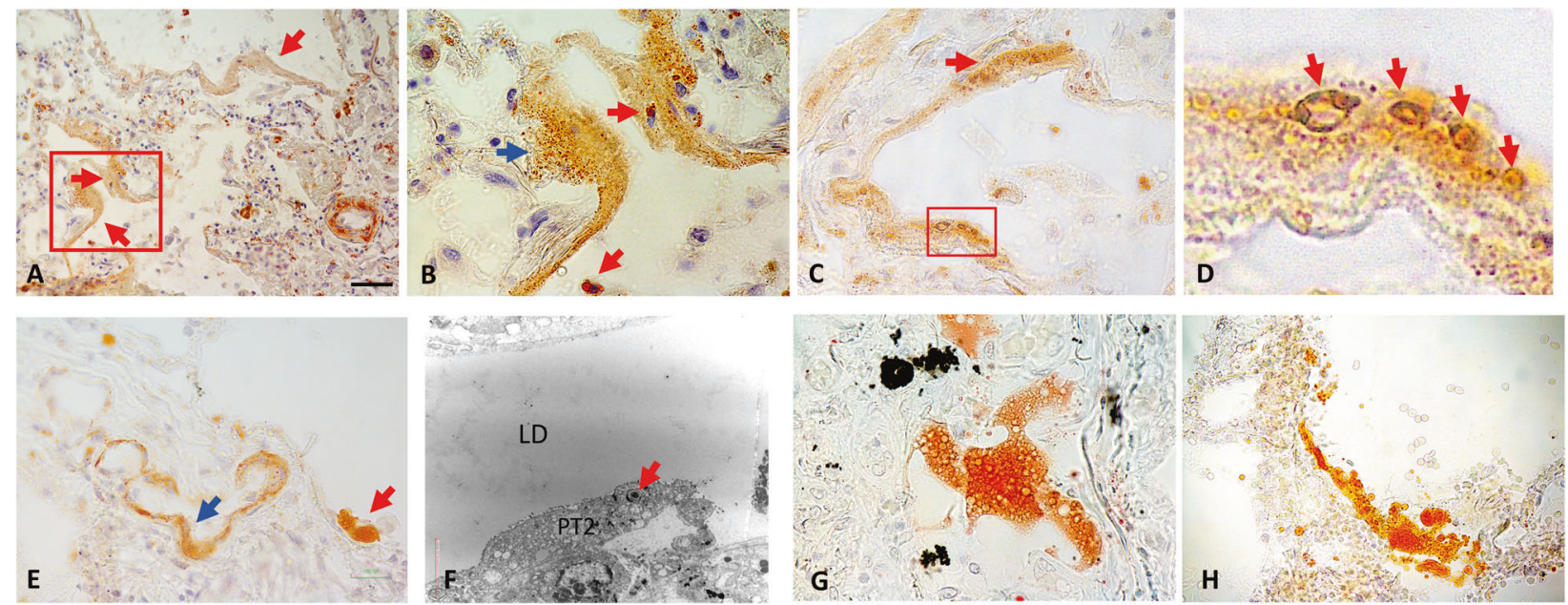

Fig. 4 Oil Red O-stained lung of subjects with COVID-19 showing hyaline membranes morphology and composition. A Light microscopy (LM): hyaline membranes lining alveolar surfaces (arrows) at low magnification. B LM: enlargement of the squared area in $\mathbf{A}$ showing the microvacuolar nature of ORO + hyaline membrane (blue arrow). Lipid-rich macrophages free in the alveolar space (red arrows) and inside hyaline membranes (red arrow). C LM: vacuolar aspect of ORO + hyaline membranes' lipids (arrow and squared area). D LM: enlargement of the squared area in C. Arrows indicate lipid vacuoles. E LM: ORO + large, free lipid vacuole lining the alveolar surface (red arrow) near a hyaline membrane (blue arrow). F TEM: free lipid droplets lining the alveolar surface composed by pneumocytes type II (PT2) with classic surfactant granules (arrow). G LM: ORO + lipid vacuole spreading on the alveolar surface (possible early stage of lipid diffusion). H LM: ORO + lipid vacuoles possibly contributing to hyaline membranes development (later stage). Scale bar: $\mathbf{A}, \mathbf{E}=50 \mu \mathrm{m}, \mathbf{B}=7 \mu \mathrm{m}, \mathbf{C}=10 \mu \mathrm{m}, \mathbf{D}=2 \mu \mathrm{m}, \mathbf{F}=$ $3 \mu \mathrm{m}, \mathbf{G}=25 \mu \mathrm{m}, \mathbf{H}=35 \mu \mathrm{m}$.

ORO-positive (Fig. 4A). The presence of HM was then confirmed by hematoxylin and eosin (H\&E), Mallory, and periodic acid-Schiff staining (data not shown). All subjects with COVID-19 presented $\mathrm{ORO}+\mathrm{HM}$ in the lungs, which were detected only in one control subject (BMI $21.3 \mathrm{~kg} / \mathrm{m}^{2}$ ) who died of pneumonia $(p<0.0001)$. Interestingly, this last subject displayed a fainted HM positivity for ORO staining, suggesting a lower lipidic presence. This finding is consistent with other reports describing $\mathrm{HM}$ in the lungs of patients with non-COVID-19-related pneumonia [7]. Importantly, $\mathrm{ORO}+$ lipid droplets and lipid-rich macrophages were often enclosed into the HM lining the alveolar surface (Fig. 4B-D). Several aspects suggesting a direct role of embolic fat in HM formation were observed. Specifically, free lipid droplets occupying the alveolar space and lining and spreading on the alveolar surface were observed (Fig. 4E-H). The presence of lung's HM of lipidic nature was associated with VAT inflammation ( $8.0 \pm 5.4$ vs $3.7 \pm 1.8 \mathrm{CD} 68+$ macrophages/10 adipocytes in subjects with and without HM, respectively) and exclusive of COVID-19+ cases (Supplementary Fig. 2).

Lastly, since the embolic material from abdominal visceral tissues should necessarily pass through the liver parenchyma to reach the lung, we exploited the ORO staining technique to study liver samples belonging to 9 individuals with COVID-19 and 8 control subjects. Liver autoptic samples showed focal, macrovesicular steatosis with lipid droplets of very variable size (Supplementary Fig. 3A), consistent with other studies conducted on patients with COVID-19 [34]. In particular, signs consistent with fat embolism, i.e., presence of free lipid droplets into hepatic sinusoids (Supplementary Fig. 3B) and into vessels lumen (Supplementary Fig. 3C, D), as well as clusters of lipid-rich structures in the portal vein (Supplementary Fig. 3D) were observed in the COVID-19+ group, a finding that confirmed the embolic nature of hepatic fat droplets, and that support what observed in VAT samples. In summary, 8/9 COVID-19+ with documented pulmonary fat embolism also displayed signs of hepatic fat embolism. On the other hand, we observed hepatic embolism in a high percentage of controls (6/8), possibly due to the elevated prevalence of visceral obesity among these investigated cases.

\section{DISCUSSION}

This is the first study investigating the ultrastructural features of VAT among individuals with COVID-19 and assessing lipid distribution in lungs and liver samples by histomorphology. Our data support the presence of higher local VAT inflammation and higher prevalence of fat embolism and lipidic HM formations in the lungs of subjects dead due to COVID-19 compared to control individuals dead for different reasons. In addition, our data support SARS-CoV-2 ability to infect human adipocytes in vitro.

Considering the strong association between COVID-19-related complications and obesity, especially with visceral adipose content excess $[10,11,13-16]$, the comprehension of the biological phenomenon at the basis of such association holds critical clinical implications in the era of the COVID-19 pandemic.

Our study provides the first evidence of higher local VAT inflammation among subjects with COVID-19, independently of obesity status and support an exacerbation of obesity-related inflammation by SARS-CoV-2 infection, a novel finding consistent with studies reporting higher systemic inflammation among infected patients [18]. Adipocyte inflammation is associated with cell stress, death, and lipid release in the extracellular space $[19,20,25,26]$. We hence studied adipocyte features by TEM and revealed the presence of the typical signs of cellular stress, together with prominent features of lipids spill-over from suffering adipocytes. Of note, these data are supported by a recent work showing an increased number of autoimmune antibodies against the malondialdehyde and the adipocyte-derived protein antigen (markers of lipid peroxidation and adipocytes death, respectively [35]) among subjects with COVID-19 and obesity as compared to individuals suffering from each condition independently [36]. In addition, hyperglycemia among subjects with COVID-19 was demonstrated to be strongly associated with insulin resistance and low plasma adiponectin levels [29]. The authors from the same study also demonstrated that SARS-CoV-2 could infect hamster adipose tissue, leading to reduced adiponectin production and speculated that SARS-CoV-2 infection might result in adipocyte dysfunction driving insulin resistance.

Importantly, we detected lipids in the extracellular space, inside endothelial cells, inside the capillary lumen, and extruding from 
1014

endothelial cells into the capillary lumen, all features indicative of fat embolism. Although virus-like structures were evidenced by TEM in the same VAT depots, the lack of SARS-CoV-2 detection by qPCR did not allow us to conclude that such inflammation, cellular stress, and death were all related to the presence of the virus. It is, in fact, possible that the described VAT features were secondary to the systemic inflammation induced by COVID-19 or due to the presence of different viruses within the depot. On the other side, we demonstrated that SARS-CoV-2 could infect human adipocytes even though neither adipocytes nor adipocyte progenitors gathered all of the known molecular requirements for the virus entry (e.g., expression of all known virus proteases and receptors). This set of data is in part consistent with other findings and suggests that additional, not yet characterized, receptors and proteases may be exploited for this purpose [16, 37]. Puray-Chavez et al. in fact indicated that human H522 lung adenocarcinoma cells are permissive to SARS-CoV-2 infection despite complete ACE2 absence and that virus entry is dependent on heparan sulfate in this cell line [37]. Importantly, despite being the first SARS-CoV-2 targets, olfactory and respiratory epithelial cells express low ACE2 protein levels [38]. For these reasons, additional co-factors facilitating the virus-host cell interaction (e.g., NEUROPILIN-1 $[39,40])$ may be critical for the infection of tissues expressing low ACE2 levels. In our study, BASIGIN receptor and FURIN protease were highly expressed in differentiated hMADS and could be exploited for SARS-CoV-2 infection. However, it should be noted that, although FURIN critical role in mediating SARS-CoV-2 infection is widely accepted and seem to be of relevance in patients with type 2 diabetes where the protease is highly expressed [41], the role of BASIGIN has been recently questioned [42].

Given the widespread presence of lipid droplets in the capillary lumen of VAT and our preliminary data [23], we studied lipid distribution in lung and liver samples and confirmed the presence of fat embolism. Interestingly, we noticed similar lipid-like structures also in lung's images from other reports on patients with COVID-19 [5, 6, 43], which is why we believe it is worth performing further in-depth analyses on available samples.

Fat embolism in the lungs was not exclusive to, but more prevalent among subjects with COVID-19; it was in fact also detected among subjects with obesity independently of SARSCoV-2 infection. These data are not surprising given that adipocyte death and release of lipids are both phenomena occurring in obesity $[19,25,26]$. This finding provides the first evidence pointing out fat embolism as a complication of obesity (and obesity plus type 2 diabetes), determined by adipocyte death and possibly exacerbated by the COVID-19-induced inflammatory status. Importantly, studying lung's lipid accumulation, we unexpectedly revealed the presence of lipidic $\mathrm{HM}$, a formation strongly contributing/associated to COVID-19-related interstitial fibrosis and pneumonia [6]. HM were present in all patients with COVID-19 and in only one control who died for pneumonia, a finding consistent with other reports describing HM presence in this latter disease [7]. Our histomorphologic assessment revealed several aspects indicative of a direct role of embolic fat in HM formation. Consistently, the presence of lung $\mathrm{HM}$ of lipidic nature (in the lungs) was associated with VAT inflammation.

Our findings on intestinal and liver fat embolism strongly support the embolic nature of lipid droplets in the lungs. As the portal system drains venous blood from most abdominal fat depots to the liver, the embolic fat originated in the VAT necessarily pass through the liver to reach other organs. The unequivocal presence of lipid droplets into sinusoids and liver veins supports the fat embolic production by abdominal fat.

In summary, in our case series, although fat embolism may be present in obesity and type 2 diabetes independently of COVID19 , the embolic lipidic material could contribute to the formation of HM only in the case of COVID-19-related pneumonia. This novel finding holds critical clinical implications and deserves further investigation. Furthermore, these data provide insights into HM nature, as their formation process has not been characterized yet [44]. Additional studies investigating the HM nature of non-COVID19-related pneumonia are required to detail such histopathological features.

Collectively our data reveal higher local VAT inflammation in subjects with COVID-19 and SARS-CoV-2 ability to infect human adipocytes. In addition, we provide the first evidence that supports the fat embolism as a complication of obesity, likely determined by adipocyte death and exacerbated by the COVID19-induced inflammatory status. Lastly, we reveal for the first time the presence of lung's lipidic HM among all infected subjects, a novel COVID-19-related histopathological feature associated with VAT inflammation and fat embolism. Consistently, fat embolism displays similar signs and symptoms as observed in COVID-19, in line with a recently published case report [45]. When fat embolism and COVID-19 are suspected, differential diagnosis is critical for proper patient care. Based on our findings, the assessment of fat embolism symptoms is mandatory in the context of the COVID-19 pandemic, especially among patients with pulmonary symptoms, obesity, and high waist circumference, last two of which are recognized as signs of high visceral adipose accumulation. Such complex clinical status should be therefore adequately assessed and properly addressed. Our data hold critical clinical implications in the context of obesity and COVID-19 pandemics and need to be confirmed by additional studies with larger sample size.

\section{MATERIALS AND METHODS Study approval}

We followed the report "Research ethics during COVID-19 pandemic: observational, and in particular, epidemiological studies" published by the Italian Istituto Superiore di Sanità on May 2020 (Rapporto ISS COVID-19, n. 47/2020) [46]. Given that our study was observational (cross-sectional, case-control) in nature, and it was conducted on autoptic specimens and did not entail neither an intervention, nor the collection of subject's sensitive information, we have not obtained an informed consent. Our study did not entail any physical risk for the subjects. In Italy, the evaluation of non-pharmacological observational studies is not governed by the same normative references provided for the evaluation of clinical trials and observational studies concerning drugs. Furthermore, as reported in the above report [46] in the section dedicated to our type of study in conditions of pandemic and therefore of high risk for the communities, some administrative steps may be abolished. Therefore, our Institutional Review Board does not require ethical approval for studies conducted on autoptic specimens and not collecting personal or sensitive data.

\section{Study subjects and tissue sampling}

Autoptic lung, liver, and VAT samples of 49 subjects were collected at the Department of Legal Medicine of the Ospedali Riuniti of Ancona between March 2020 and May 2021. Twenty-four subjects were affected by COVID-19, while the remaining 25 were not and died for different reasons. SARS-CoV-2 infection was assessed in all subjects by RT-PCR tests on a nasopharyngeal swab. Subjects were included in the analyses only if their lung's samples were well preserved such that a high-quality histological assessment could be performed. We hence analyzed 19 COVID-19+ cases and 23 controls. Among the studied subjects, 15 had documented respiratory conditions, i.e., pneumonia, dyspnoea, respiratory distress (10 COVID-19+ and 5 controls), 15 had documented hypertension (7 COVID-19+ and 8 controls), 11 had type 2 diabetes (6 COVID-19+ and 5 controls) and 10 suffered from cardiovascular diseases (2 COVID-19+ and 8 controls). VAT was sampled from the omentum and mesentery region. Lungs were extensively sampled across central and peripheral regions of each lobe bilaterally. A median of seven tissue blocks (range five to nine) were taken from each lung. Liver samples were collected from the right and left lobes.

Samples were sliced into different pieces to be studied by LM and transmission electron microscopy (TEM). A comprehensive methodological description for such methodologies has been described elsewhere [47]. 


\section{Immunohistochemistry and morphometric analyses}

The collected visceral (omental) adipose tissue, lung and liver autopsies were fixed overnight at $4{ }^{\circ} \mathrm{C}$ in $4 \%$ paraformaldehyde. Samples were then embedded in paraffin to be studied by LM and to perform immunohistochemistry and morphometric analyses. For each sample, $3 \mu \mathrm{m}$ paraffin sections were obtained and used for immunohistochemical analyses. A comprehensive description of the protocol has been described elsewhere [47]. To detect the presence of CD68+ macrophages in VAT samples, we used CD68 (Dako \#M0814; dilution 1:200; antigen retrieval method by citrate buffer pH6) antibody. To study SARS-CoV-2 presence in VAT, we used the SARS-CoV-2 nucleocapsid (Invitrogen \#MA-17404) and spike protein (Sino Biological \#40150-T62) antibodies at different dilutions. The same antibodies were used to detect the virus on infected VeroE6 at dilution: 1:1000 for nucleocapsid protein and 1:100 for the spike protein. Negative control in which primary antibody was omitted were always included in each set of reactions to assess antibody specificity. Tissue sections were observed with a Nikon Eclipse E800 light microscope. For morphometric purposes, for each paraffin section, ten digital images were acquired at $\times 20$ magnification with a Nikon DXM 1220 camera. CD68+ macrophages widespread in VAT parenchyma and organized to form CLS were counted in all images. For each subject, the number of total macrophages and the density of $\mathrm{CLS} / 10^{4}$ adipocytes were counted with the ImageJ morphometric program (RRID:SCR_003070). Adipocytes' area was measured in all patients by counting 100 adipocytes for each paraffin tissue section using ImageJ.

\section{Histochemical staining}

For ORO staining, samples were cryoprotected in 30\% sucrose overnight, embedded in the optimal cutting temperature compound medium, and then sliced to obtain $7 \mu \mathrm{m}$ thick cryosections by Leica CM1900 cryostat (Vienna, Austria). ORO staining was then performed on lungs [43] and liver $(n=9)$ cryosections. In brief, dried cryosections were first placed in $60 \%$ isopropanol, then in filtrated ORO working solution $(15 \mathrm{~min}$ at room temperature) and briefly washed again in $60 \%$ isopropanol and lastly in $\mathrm{H}_{2} \mathrm{O}$. Tissue slices were then counterstained with hematoxylin and covered with a cover-slip using Vectashield mounting medium (Vector Laboratories). Lung and liver tissues organization and morphology were also studied by H\&E staining on paraffin sections. Lung's HM presence and characterization were performed on paraffin sections by $\mathrm{H} \& \mathrm{E}$, periodic acid-Schiff and Masson trichome staining.

\section{Transmission electron microscopy}

For ultrastructural analyses, 3-mm thick VAT $(n=4)$, lung $(n=8)$, and liver $(n=1)$ samples were further fixed in $2 \%$ glutaraldehyde- $2 \%$ paraformaldehyde in $0.1 \mathrm{M}$ phosphate buffer $(\mathrm{pH}$ 7.4) and post-fixed in Osmium Tetroxide $1 \%$ then embedded in epoxy resin for TEM studies as described elsewhere [47]. Cell pellets from the in vitro studies were similarly fixed in $2 \%$ glutaraldehyde- $2 \%$ paraformaldehyde in $0.1 \mathrm{M}$ phosphate buffer $(\mathrm{pH}$ 7.4) for $1 \mathrm{~h}$ at room temperature and then embedded in epoxy resin. An MT-X ultratome (RMC; Tucson) was used to obtain ultrathin sections $(\sim 70 \mathrm{~nm})$. Ultrastructural characterization was performed on all samples using a CM10 Philips transmission electron microscope (Philips, Eindhoven, The Netherlands, http://www.usa.philips.com).

\section{SARS-CoV-2 infection in VeroE6}

VeroE6 cells were cultured in Dulbecco's modified Eagle medium (Euroclone, Milano, Italy), supplemented with $10 \%$ fetal calf serum (FCS Euroclone) and antibiotics/antimycotic $(100 \mathrm{U} / \mathrm{ml}$ penicillin, $100 \mu \mathrm{g} / \mathrm{ml}$ streptomycin, $0.25 \mathrm{\mu g} / \mathrm{ml}$ amphotericin B) at $37^{\circ} \mathrm{C}_{\text {, }} 5 \% \mathrm{CO}_{2}$ in a humidified atmosphere (90\%), as described previously [48]. Cells were maintained in $75 \mathrm{~cm}^{2}$ tissue culture flasks. The day before infection, a confluent monolayer was trypsinized, and $1.5 \times 10^{6}$ cells were seeded in every eight flasks $\left(25 \mathrm{~cm}^{2}\right)$. Confluent monolayers were infected with SARS-CoV-2 (78952 isolates, accession no. MT483867) [49] at a multiplicity of infection of $3.29 \cdot 10^{5}$. After $2 \mathrm{~h}$ of incubation, the medium containing the inoculum was removed, the cells were washed twice, and fresh medium was added, which was collected after $6,12,24$, and $48 \mathrm{~h}$ for viral genome quantification and replaced with $2 \mathrm{ml}$ of fresh culture medium to allow scraping of the infected monolayer. Uninfected cell monolayer controls were treated as infected ones. Cell suspensions $(2 \mathrm{ml})$ were subsequently centrifuged at $800 \mathrm{rpm}$ for $5 \mathrm{~min}$. Aliquots of infected supernatants, collected as above, were analyzed using RT-qPCR assay as described elsewhere [49]. Briefly, $5 \mu \mathrm{l}$ of RNA extracted from $140 \mu$ l of infected supernatants were run together with a calibration curve, obtained from ten-fold dilutions of a standard plasmid certified and quantified by a supplier (2019-nCoV Positive Control, nCoVPC, 85 IDT) and negative control, applying a protocol described by CDC (https://www.fda.gov/ media/134922/download).

\section{In vitro studies on hMADS}

Ethical approval. Human adipocytes progenitors -Aps- (hMADS cells) were isolated from adipose tissue, as surgical scraps from a surgical specimen of various surgeries of young donors, with the informed consent of the parents. All methods were approved and performed following the guidelines and regulations of the Centre Hospitalier Universitaire de Nice Review Board.

Cell differentiation. hMADS cells were maintained and differentiated as previously described [50]. They will be further referred to as hMADS adipocytes. They were routinely tested for the absence of mycoplasma. Treatments and biological assays were carried out in duplicates on control or differentiated hMADS cells from days 4 to 18 .

Gene expression analysis. Total RNA was extracted using the TRI-Reagent kit (Euromedex, Soufflweyersheim, France) and reverse transcription (RT) was performed using MMLV reverse transcriptase (Promega, Charbonnieres, France), as recommended by the manufacturers. All primer sequences are described in the Supplementary section. Real-time PCR assays were run on an ABI Prism One-step real-time PCR machine (Applied Biosystems, Courtaboeuf, France). Normalization was performed using $36 B 4$ as a reference gene. Quantification was performed using the comparative $\mathrm{Ct}$ method. The results are shown as mean \pm standard error of the mean (SEM), with the number of experiments indicated. Statistical significance was determined by $t$-tests BiostaTGV (INSERM and Sorbonne University, PARIS, France). Probability values $<0.05$ were considered statistically significant and are marked with a single asterisk, $<0.01$ with double asterisks and $<0.001$ with triple asterisks. Sequences for the primers used in this study ACE2 (FW 5'- AGAACCCTGGACCCTAGCAT -3'; REV 5'- AG TCGGTACTCCATCCCACA -3'); BASIGIN (FW: 5'- CAGAGTGAAGGCCGTGAAG T -3'; REV: 5'- ACTCTGACTTGCAGACCAGC -3'); NRP1 (FW: 5'- GGGGCTCTCAC AAGACCTTC -3'; REV: 5'- GATCCTGAATGGGTCCCGTC -3'); CSTL (FW: 5'- CTG GTGGTTGGCTACGGATT -3'; REV: 5'- CTCCGGTCTTTGGCCATCTT -3'); FURIN (FW: 5'-CTACAGCAGTGGCAACCAGA-3'; REV: 5'- TGTGAGACTCCGTGCACT TC-3'); $36 B 4$ (FW: 5'- CTACAACCCTGAAGAAGTGCTTG -3'; REV: 5'- CAATC TGCAGACAGACACTGG -3'); DPP4 (SINO biologicals Inc. \#HP100-649 (Eschborn, Germany)

hMADS SARS-CoV-2 infection. hMADS and hMADS adipocytes cells were infected with a viral stock of SARS-CoV-2 (EPI_ISL_417491), at a 50\% Tissue Culture Infectious Dose $\left(\mathrm{TCID}_{50}\right)$ of $2000 \mathrm{TCID}_{50} / \mathrm{ml}$ for $2 \mathrm{~h}$ at a temperature of $37^{\circ} \mathrm{C}$. Following incubation, the medium containing the inoculum was removed, the cells were washed twice, and the medium was supplemented with different specific compounds. Supernatants were collected at 24, $48,72,96 \mathrm{~h}$ for viral genome quantification, and medium renewal was performed at each sampling time. Uninfected cell monolayer controls were treated as the infected ones. Supernatants, collected as above, and cell pellets, collected at $96 \mathrm{~h}$ post-infection, were analyzed using RT-qPCR as described in the VeroE6 cell section.

Cell viability assay (MTT assay). The effect of SARS-CoV-2 infection on cell viability of hMADS adipocytes was measured using the metabolic dye [4,5dimethylthiazol-2-yl]-2,5-diphenyl tetrazolium (MTT) (Sigma, St. Louis, MO, USA). Briefly, hMADS cells were seeded in 96-well plates at a density of 4500 cells $/ \mathrm{cm}^{2}$, differentiated and then infected with the viral stock of SARS-CoV-2 for $2 \mathrm{~h}$ at $37^{\circ} \mathrm{C}$. Following the incubation with the virus, cells were placed in supplemented medium. Time-course analyses of cell survival were determined at $24,48,72$, and $96 \mathrm{~h}$. After the incubation period, the media were replaced with $100 \mu \mathrm{MTT}(0.5 \mathrm{mg} / \mathrm{ml})$, dissolved in PBS and incubated for $3 \mathrm{~h}$. MTT-containing medium was removed, and $100 \mu \mathrm{l}$ of dimethyl sulfoxide was added to dissolve formazan crystals formed by live cells. Absorbance was subsequently measured at $570 \mathrm{~nm}$ using a BioTek Synergy HTX microplate reader (BioTek, Winooski, VT, USA). Results were expressed as percentages of viable cells relative to uninfected controls.

Nuclear morphology analyses. Alterations in nuclear morphology were determined by assessment of nuclear staining using fluorescent stains and fluorescent microscopy [51]. 
For these experiments, hMADS adipocytes were differentiated in 2-well Lab-Tek Chamber Slides (Nalge Nunc International, Naperville, IL, USA), washed with PBS pH 7.4 and fixed with $10 \%$ paraformaldehyde in PBS for 10 min at RT. After washing with PBS, nuclear staining was performed with Hoechst. Finally, cells were airdried and cover-slipped using Vectashield mounting medium (Vector Laboratories, Burlingame, CA, USA) and analyzed by fluorescent microscopy. The number of altered nuclei were counted (in the field displaying nuclear fragmentation and nuclear condensation) and divided by the total number of nuclei multiplied by 100 . Observations were carried out by Lucia IMAGE 4.82, Laboratory Investigations Morphometric Analyses.

Lipid droplet size $\left(\mu \mathrm{m}^{2}\right)$ was measured in SARS-CoV-2-infected hMADS adipocytes and untreated controls. For this purpose, we used a drawing tablet and a morphometric program (Nikon LUCIA IMAGE, Laboratory Imaging, version 4.61; Praha, Czech Republic). hMADS adipocytes were examined with a Nikon Eclipse Ti-S inverted light microscope (Nikon Instruments S.p.A, Calenzano, Italy), and digital images were captured at $\times 20$ with a Nikon DS-L2 camera (Nikon Instruments S.p.A, Calenzano, Italy). Five random fields were analyzed, at least 1700 lipid droplets were measured for each sample, and the difference between infected and non-infected cells was assessed by unpaired $t$-test. Similarly, the quantitative assessment of the material extruded from the hMADS was calculated using the same microscope and software and expressed as the number of vacuoles extruded from the cells on the total cell amount.

\section{Statistical analysis}

Between-group comparisons for linear and categorical variables were determined by unpaired two-tailed Student's $t$ test and Chi-square test, respectively. Group differences were considered significant when $p<0.05$. Data in graphs are expressed as mean \pm SEM. Statistical analyses were performed with Prism 6.0 (GraphPad Software Inc., La Jolla, CA) and IBM SPSS Statistics Data Editor (v.24).

\section{REFERENCES}

1. Medicine JHU. Coronavirus resource center. 2021. https://coronavirus.jhu.edu/ map.html.

2. Mahendra M, Nuchin A, Kumar R, Shreedhar S, Mahesh PA. Predictors of mortality in patients with severe COVID-19 pneumonia-a retrospective study. Adv Respir Med. 2021;89:135-44.

3. Huang C, Wang Y, Li X, Ren L, Zhao J, Hu Y, et al. Clinical features of patients infected with 2019 novel coronavirus in Wuhan, China. Lancet. 2020;395:497-506.

4. Wadman M, Couzin-Frankel J, Kaiser J, Matacic C. A rampage through the body. Science. 2020;368:356-60.

5. Xu Z, Shi L, Wang Y, Zhang J, Huang L, Zhang C, et al. Pathological findings of COVID-19 associated with acute respiratory distress syndrome. Lancet Respir Med. 2020;8:420-2.

6. Bradley BT, Maioli H, Johnston R, Chaudhry I, Fink SL, Xu H, et al. Histopathology and ultrastructural findings of fatal COVID-19 infections in Washington State: a case series. Lancet. 2020;396:320-32.

7. Schneider JL, Rowe JH, Garcia-de-Alba C, Kim CF, Sharpe AH, Haigis MC. The aging lung: physiology, disease, and immunity. Cell. 2021;184:1990-2019.

8. Stefan N, Birkenfeld AL, Schulze MB. Global pandemics interconnected-obesity, impaired metabolic health and COVID-19. Nat Rev Endocrinol. 2021;17:135-49.

9. O'Hearn M, Liu J, Cudhea F, Micha R, Mozaffarian D. Coronavirus Disease 2019 hospitalizations attributable to cardiometabolic conditions in the United States: a comparative risk assessment analysis. J Am Heart Assoc. 2021;10:e019259.

10. Williamson EJ, Walker AJ, Bhaskaran $\mathrm{K}$, Bacon S, Bates $C$, Morton $C E$, et al. Factors associated with COVID-19-related death using OpenSAFELY. Nature. 2020;584:430-6.

11. Onder G, Palmieri L, Vanacore N, Giuliano M, Brusaferro S,Italian National Institute of Health C-MG. Nonrespiratory complications and obesity in patients dying with COVID-19 in Italy. Obesity. 2021;29:20-3.

12. Locke $A E$, Kahali $B$, Berndt SI, Justice $A E$, Pers TH, Day FR, et al. Genetic studies of body mass index yield new insights for obesity biology. Nature. 2015;518:197-206.

13. Battisti S, Pedone C, Napoli N, Russo E, Agnoletti V, Nigra SG, et al. Computed tomography highlights increased visceral adiposity associated with critical illness in COVID-19. Diabetes Care. 2020;43:e129-30.

14. Watanabe $M$, Caruso D, Tuccinardi D, Risi R, Zerunian M, Polici M, et al. Visceral fat shows the strongest association with the need of intensive care in patients with COVID-19. Metabolism. 2020;111:154319.

15. Petersen A, Bressem K, Albrecht J, Thiess HM, Vahldiek J, Hamm B, et al. The role of visceral adiposity in the severity of COVID-19: Highlights from a unicenter cross-sectional pilot study in Germany. Metabolism. 2020;110:154317.

16. Drucker DJ. Diabetes, obesity, metabolism, and SARS-CoV-2 infection: the end of the beginning. Cell Metab. 2021;33:479-98.
17. Kompaniyets L, Goodman AB, Belay B, Freedman DS, Sucosky MS, Lange SJ, et al. Body mass index and risk for COVID-19-related hospitalization, intensive care unit admission, invasive mechanical ventilation, and death-United States, MarchDecember 2020. Morb Mortal Wkly Rep. 2021;70:355-61.

18. Morys F, Dagher A. Poor metabolic health increases COVID-19-related mortality in the UK biobank sample. Front Endocrinol. 2021;12:652765.

19. Cinti S, Mitchell G, Barbatelli G, Murano I, Ceresi E, Faloia E, et al. Adipocyte death defines macrophage localization and function in adipose tissue of obese mice and humans. J Lipid Res. 2005;46:2347-55.

20. Hotamisligil GS. Inflammation, metaflammation and immunometabolic disorders. Nature. 2017;542:177-85.

21. Colleluori G, Villareal DT. Aging, obesity, sarcopenia and the effect of diet and exercise intervention. Exp Gerontol. 2021;155:111561.

22. Gupte M, Boustany-Kari CM, Bharadwaj K, Police S, Thatcher S, Gong MC, et al. ACE2 is expressed in mouse adipocytes and regulated by a high-fat diet. Am J Physiol Regul Integr Comp Physiol. 2008;295:R781-8.

23. Cinti S, Graciotti L, Giordano A, Valerio A, Nisoli E. COVID-19 and fat embolism: a hypothesis to explain the severe clinical outcome in people with obesity. Int J Obes. 2020;44:1800-2.

24. Shin J, Toyoda S, Nishitani S, Fukuhara A, Kita S, Otsuki M, et al. Possible involvement of adipose tissue in patients with older age, obesity, and diabetes with Coronavirus SARS-CoV-2 infection (COVID-19) via GRP78 (BIP/HSPA5): significance of hyperinsulinemia management in COVID-19. Diabetes. 2021;70:2745-2755.

25. Murano I, Barbatelli G, Parisani V, Latini C, Muzzonigro G, Castellucci M, et al. Dead adipocytes, detected as crown-like structures, are prevalent in visceral fat depots of genetically obese mice. J Lipid Res. 2008;49:1562-8.

26. Camastra S, Vitali A, Anselmino M, Gastaldelli A, Bellini R, Berta R, et al. Muscle and adipose tissue morphology, insulin sensitivity and beta-cell function in diabetic and nondiabetic obese patients: effects of bariatric surgery. Sci Rep. 2017;7:9007.

27. Meng $Y$, Zhang $M$, Ling $H$, Huang $S$, Miao $Q, Y u$, et al. Nontraumatic multipleorgan fat embolism: an autopsy case and review of literature. Am J Forensic Med Pathol. 2020;41:131-4.

28. Hirschbuhl K, Dintner S, Beer M, Wylezich C, Schlegel J, Delbridge C, et al. Viral mapping in COVID-19 deceased in the Augsburg autopsy series of the first wave: a multiorgan and multimethodological approach. PLoS ONE. 2021;16:e0254872.

29. Reiterer M, Rajan M, Gomez-Banoy N, Lau JD, Gomez-Escobar LG, Ma L, et al. Hyperglycemia in acute COVID-19 is characterized by insulin resistance and adipose tissue infectivity by SARS-CoV-2. Cell Metab. 2021;33:2174-88 e5.

30. Goldsmith CS, Tatti KM, Ksiazek TG, Rollin PE, Comer JA, Lee WW, et al. Ultrastructural characterization of SARS coronavirus. Emerg Infect Dis. 2004;10:320-6.

31. Ghadially F. Ultrastructural pathology of the cell and matrix. 1997 (ButterworthHeinemann, Boston).

32. Giordano A, Murano I, Mondini E, Perugini J, Smorlesi A, Severi I, et al. Obese adipocytes show ultrastructural features of stressed cells and die of pyroptosis. J Lipid Res. 2013;54:2423-36.

33. Perlman S, Netland J. Coronaviruses post-SARS: update on replication and pathogenesis. Nat Rev Microbiol. 2009;7:439-50.

34. Lagana SM, Kudose S, luga AC, Lee MJ, Fazlollahi L, Remotti HE, et al. Hepatic pathology in patients dying of COVID-19: a series of 40 cases including clinical, histologic, and virologic data. Mod Pathol. 2020;33:2147-55.

35. Frasca D, Diaz A, Romero M, Garcia D, Jayram D, Thaller $S$, et al. Identification and characterization of adipose tissue-derived human antibodies with "Anti-self" specificity. Front Immunol. 2020;11:392.

36. Frasca D, Reidy L, Romero M, Diaz A, Cray C, Kahl K, et al. The majority of SARS-CoV2-specific antibodies in COVID-19 patients with obesity are autoimmune and not neutralizing. Int J Obes. 2021;1-6. https://doi.org/10.1038/s41366-021-01016-9.

37. Puray-Chavez M, LaPak KM, Schrank TP, Elliott JL, Bhatt DP, Agajanian MJ, et al. Systematic analysis of SARS-CoV-2 infection of an ACE2-negative human airway cell. Cell Rep. 2021;36:109364.

38. Hikmet $F$, Mear L, Edvinsson A, Micke P, Uhlen $M$, Lindskog $C$. The protein expression profile of ACE2 in human tissues. Mol Syst Biol. 2020;16:e9610.

39. Cantuti-Castelvetri L, Ojha R, Pedro LD, Djannatian M, Franz J, Kuivanen S, et al. Neuropilin-1 facilitates SARS-CoV-2 cell entry and infectivity. Science. 2020;370:856-60.

40. Giordano A, Cesari P, Capparuccia L, Castellucci M, Cinti S. Sema3A and neuropilin-1 expression and distribution in rat white adipose tissue. J Neurocytol. 2003;32:345-52.

41. Ganesan SK, Venkatratnam P, Mahendra J, Devarajan N. Increased mortality of COVID19 infected diabetes patients: role of furin proteases. Int J Obes. 2020;44:2486-8.

42. Ragotte RJ, Pulido D, Donnellan FR, Hill ML, Gorini G, Davies H, et al. Human basigin (CD147) does not directly interact with SARS-CoV-2 spike glycoprotein. mSphere. 2021;6:e0064721.

43. Konopka KE, Nguyen T, Jentzen JM, Rayes O, Schmidt CJ, Wilson AM, et al. Diffuse alveolar damage (DAD) resulting from coronavirus disease 2019 Infection is Morphologically Indistinguishable from Other Causes of DAD. Histopathology. 2020;77:570-8. 
44. Wellman TJ, de Prost N, Tucci M, Winkler T, Baron RM, Filipczak P, et al. Lung metabolic activation as an early biomarker of acute respiratory distress syndrome and local gene expression heterogeneity. Anesthesiology. 2016;125:992-1004.

45. Alexa AL, Onutu AH. Fat embolism syndrome mimicking a COVID-19 infection Case Rep Crit Care. 2021;2021:5519812.

46. ISS. Istituto Superiore di Sanità-Research ethics during the COVID-19 pandemic: observational and, in particular, epidemiological studies. 2020.

47. Cinti S, Zingaretti MC, Cancello R, Ceresi E, Ferrara P. Morphologic techniques for the study of brown adipose tissue and white adipose tissue. Methods Mol Biol. 2001;155:21-51.

48. de Wilde AH, Raj VS, Oudshoorn D, Bestebroer TM, van Nieuwkoop S, Limpens R, et al. MERS-coronavirus replication induces severe in vitro cytopathology and is strongly inhibited by cyclosporin A or interferon-alpha treatment. J Gen Virol. 2013;94:1749-60.

49. Alessandrini F, Caucci S, Onofri V, Melchionda F, Tagliabracci A, Bagnarelli $P$, et al. Evaluation of the ion AmpliSeq SARS-CoV-2 research panel by massive parallel sequencing. Genes. 2020;11:929-941.

50. Rodriguez AM, Elabd C, Amri EZ, Ailhaud G, Dani C. The human adipose tissue is a source of multipotent stem cells. Biochimie. 2005;87:125-8.

51. Cummings BS, Schnellmann RG. Measurement of cell death in mammalian cells. Curr Protoc Pharmacol. 2004.

52. Colleluori G, Graciotti L, Pesaresi M, Di Vincenzo A, Perugini J, Di Mercurio E, et al. Visceral fat inflammation and fat embolism are associated with lung's lipidic hyaline membranes in COVID-19 patients. bioRxiv. 2021. https://doi.org/10.1101/ 2021.10.30.466586.

\section{ACKNOWLEDGEMENTS}

This study was funded by Fondo Integrativo Speciale per la Ricerca from the Italian Ministry of University and Research; grant number: FISR2020IP_05217, and supported by Progetti di Rilevante Interesse Nazionale (PRIN 2017, \#2017L8Z2) and by Cariplo Foundation to EN (grant 2016-1006). Results from this study were made available on November 1st 2021 on bioRxiv [52]

\section{AUTHOR CONTRIBUTIONS}

GC, LG, MP, AG, and SC: study conceptualization. GC, LG, and SC: study coordination. MP and AT: collected autoptic samples and clinical data. GC, MP, and ADV histological studies on autoptic samples and cell cultures. CMZ, LG, and SC: electron microscopy studies. JP, EDM, AL, and CD: in vitro studies on hMADS. SC, PB, and SM: SARS-CoV-2 infection for the in vitro studies. GC, LG, MP, JP, EN, SM, AG, and SC: data analyses and interpretation. All authors approved the final version of the manuscript and take responsibility for its content.

\section{COMPETING INTERESTS}

The authors declare no competing interests.

\section{ADDITIONAL INFORMATION}

Supplementary information The online version contains supplementary material available at https://doi.org/10.1038/s41366-022-01071-w.

Correspondence and requests for materials should be addressed to Saverio Cinti.

Reprints and permission information is available at http://www.nature.com/ reprints

Publisher's note Springer Nature remains neutral with regard to jurisdictional claims in published maps and institutional affiliations. 